
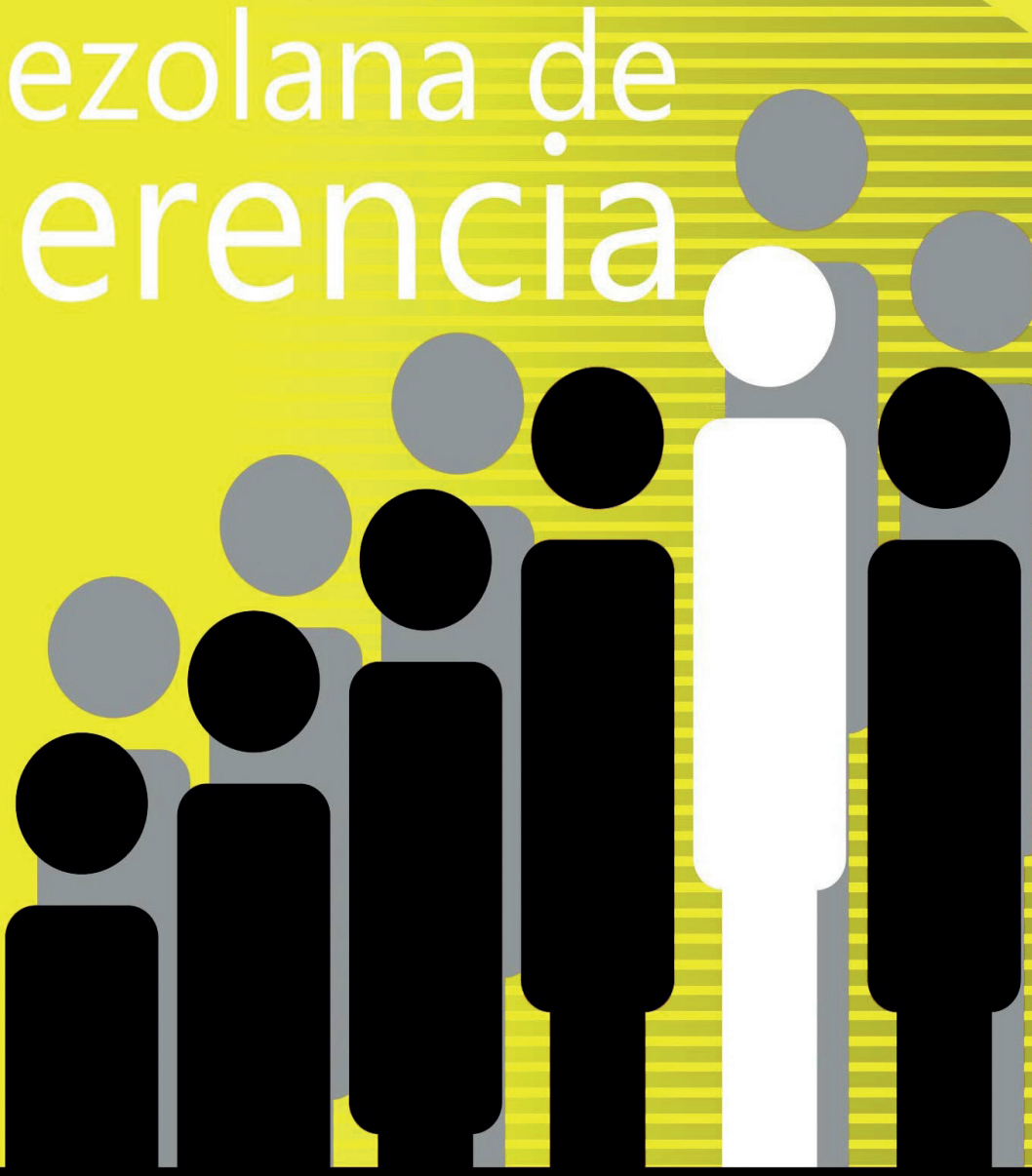


\title{
Planificación estratégica situacional: Un proceso metódico-práctico
}

\author{
Gutiérrez Silva, José Manuel ${ }^{*}$ \\ Romero Borré, Jenny \\ Hernández Fernández, Lissette \\ Vega Jaramillo, Flor Yelena ${ }^{* \star \star x}$
}

\section{Resumen}

La planificación situacional asume la crítica de la planificación tradicional, por su incapacidad de producir cambios, siendo de auto referencia del hombre de acción, que en última instancia debe resolver problemas complejos, mostrando entonces una herramienta de conducción o gobierno del proceso social en todas sus dimensiones. Bajo estas consideraciones, el presente artículo analiza el modelo metódico-práctico de la planificación estratégica situacional. Teóricamente se fundamentó en Matus (1987), Alizo (2005), Alizo (2020), Zambrano (2006), entre otros. La naturaleza de la investigación es de carácter descriptivo, con un enfoque analítico, cuya fuente de información es de tipo documental. Se evidencia luego de la discusión de los diferentes momentos por los que atraviesa el modelo (explicativo, normativo, estratégico y táctico-operacional), que este tipo de planificación toma atención en el "ser", más no en el "debe ser", siendo más que un simple proceso, en el cual se deben establecer metas y objetivos que tienen que

\section{Recibido: 15.08.20 Aceptado: 20.01.21}

Economista. Docente - Investigador adscrito al Instituto de Investigaciones "Econ. Dionisio Carruyo" de la Facultad de Ciencias Económicas y Sociales de la Universidad del Zulia (Maracaibo-Venezuela). E-mail: imgsilva30@gmail.com ORCID: https://orcid.org/0000-0001-9929-0172

* Doctora en Ciencias Humanas. Magíster en Gerencia de Empresas, Mención Finanzas. Economista. Docente e Investigadora adscrita a la Facultad de Ciencias Económicas de la Universidad de la Costa (BarranquillaColombia) y al Instituto de Investigaciones -Econ. Dionisio Carruyo- de la Facultad de Ciencias Económicas y Sociales de la Universidad del Zulia (Maracaibo-Venezuela). E-mail: jennyfrb@yahoo.com ORCID: https:// orcid.org/0000-0002-0457-6467

*** Doctora en Ciencias Humanas. Magíster en Gerencia de Empresas, Mención Finanzas. Magíster en Economía, Mención Macroeconomía y Política Económica. Economista. Docente - Investigadora de la Universidad del Atlántico (Barranquilla-Colombia). Profesora emérita de la Universidad del Zulia (MaracaiboVenezuela). E-mail: lissettehf@yahoo.es ORCID: https://orcid.org/0000-0003-0839-6057

**** Candidata a Doctora en Ciencias Económicas. Magister en Tributación y Finanzas. Economista, Mención Gestión Empresarial. Docente-Investigadora de la Facultad de Ciencias Empresariales de la Universidad Técnica de Machala (Machala-Ecuador).E-mail: yelenavega26@gmail.com 
ser cumplidos durante períodos específicos, con el propósito de alcanzar la situación futura planeada. Por ello, constituye una herramienta indispensable para cualquier tipo de organización y de ejercicio para la alta gerencia.

Palabras clave: Planificación estratégica situacional; momentos; problemas; proyecto estratégico; acción.

\title{
Situational strategic planning: A practical- methodical process
}

\begin{abstract}
Situational planning assumes the criticism of traditional planning, due to its inability to produce changes, being the self-reference of the man of action, who ultimately must solve complex problems, thus showing a tool for conducting or governing the social process in all its dimensions. Under these considerations, this article analyzes the methodical-practical model of situational strategic planning. Theoretically, it was based on Matus (1987), Alizo (2005), Alizo (2020), Zambrano (2006), among others. The nature of the research is descriptive, with an analytical approach, whose source of information is documentary. It is evident after the discussion of the different moments that the model goes through (explanatory, normative, strategic and tactical-operational), that this type of planning pays attention to the "being", but not the "must be". Being more than a simple process, in which goals and objectives must be established that have to be met during specific periods, in order to achieve the planned future situation. Therefore, it is an indispensable tool for any type of organization and exercise for senior management.
\end{abstract} action.

Key words: Situational strategic planning; moments; problems; strategic project;

\section{Introducción}

El desenvolvimiento del entorno económico, político y social que vienen enfrentando los países, ha tenido que girar en medio de la planeación de actividades, siendo imprescindible en todo tipo de espacios y organizaciones, convirtiéndose en la mejor herramienta ante la incertidumbre de lo que pueda ocurrir en un sistema. Estas y otras consideraciones son las que llevan a pensar en planificación, que apuntan según Matus (1987), al centro del concepto del plan o el resultado de los intentos por responder a las condiciones existentes como señala Basurto (2016).

Corredor (2004) comenta que la planificación es el intento deliberado de proveer o seleccionar medios, que asignándolos racionalmente conduce a los fines deseados, traduciéndose en un proceso mediante el cual se anticipa el futuro, desde el presente con base 
Gutiérrez Silva, José Manuel; Romero Borré, Jenny; Hernández Fernández, Lissette; Vega Jaramillo, Flor Yelena

Planificación estratégica situacional: Un proceso metódico-práctico

al pasado. Donde no sólo se pretende anticipar lo que viene más adelante, sino también labrar el porvenir, esto es, transformar el futuro esperado en un futuro deseado.

Sabino (2005) bajo una visión economicista, indica que planificar consiste en trazar un plan que fije los factores productivos para obtener resultados previamente definidos, reduciendo la incertidumbre al programar las actividades que se realizarán. Gutiérrez et al, (2016) exponen que es el proceso de establecer objetivos y escoger el medio más apropiado para el logro de los mismos, antes de emprender la acción, desarrollando una serie de procedimientos y operaciones que prevén el futuro. En ese marco, la planificación se fue convirtiendo en un cuadro de referencia importante para el futuro de los países, donde el Estado toma en sus manos esta herramienta metódica en la búsqueda de desarrollo para sus naciones (Gutiérrez y Colina, 2013).

Martner y Máttar (2012) señalan que en gran parte de los países de América Latina, a mediados del siglo $X X$, el Estado era el actor esencial de la planificación, a través de la construcción de políticas direccionales que pretendían encaminar las circunstancias económicas a unas condiciones que favorecieran a todos los miembros de la sociedad. No obstante, Zambrano (2006) afirma que el Estado con sus recursos ciertamente puede avanzar significativamente en un proceso de planificación, o un gerente en la cabeza de una organización, pero independientemente del sujeto que coordine el plan, lo realmente importante es que este fije y materialice los ideales meta, teniendo en cuenta interrogantes como: ¿Dónde estamos?, ¿A dónde vamos?, ¿Qué hacer?, ¿Cómo llegamos allá?

Se puede sintetizar entonces, que detrás de la conceptualización del término planificación, se encuentra como base fundamental el deseo de poder alcanzar una necesidad humana, económica y social, que conlleve a revertir situaciones presentes en un sistema encaminado a determinar la acción futura. Alizo (2020) afirma que es sinónimo de planeamiento y conforma todo un método para resolver los problemas sociales que se materializan en obras concretas, donde convergen múltiples disciplinas que abordan la realidad de manera holística e integral y de forma sistemática cumple etapas y actividades establecidas.

Direccionar el éxito deseado, estará conformado por acciones, recursos, capacidad, perseverancia, competitividad e intención de alcanzar lo propuesto. Sin embargo, hay que tener en cuenta que no es tarea fácil, lograr todo lo planeado implica hacer frente a variables que condicionan en muchos de los casos, las actividades previstas. Por tanto, se debe conocer realmente la tipología a planificar, donde la predicción o pronóstico son elementos fundamentales.

Bajo estas consideraciones, el presente artículo hace una reflexión teórica sobre el proceso de planificación estratégica situacional, abordada por Matus (1931-1998), destacando la metodología de esta variable, aplicable a la gestión pública y privada. Esto con la intención de contar con teoría sistematizada vigente y necesaria dado los acontecimientos que viven numerosos gobiernos y organizaciones. 


\section{Planificación estratégica situacional: la visión de un proceso metódico-práctico}

Nace como alternativa y oposición a la planificación táctica, tradicional o normativa, que sólo toma atención en el 'debe ser' más no en el 'ser', por lo que es mucho más que un simple proceso (Matus, 1987), en el cual se deben establecer metas y objetivos que tienen que ser cumplidos durante períodos específicos, con el propósito de alcanzar la situación futura planeada. Por ello, constituye una herramienta indispensable para cualquier tipo de organización y de ejercicio para la alta gerencia (Gutiérrez et al, 2016).

Matus (1987) expone que la planificación estratégica situacional (PES) asume la crítica de la planificación tradicional, la coloca de auto referencia para la resolución de problemas complejos, mostrando entonces una herramienta de conducción o gobierno del proceso social en todas sus dimensiones. Basurto (2016) aclara que está integrada de un enfoque metodológico, debido al desarrollo de categorías centrales que con sus interrelaciones, son capaces de representar las relaciones direccionales y causales de una tipología de problemas que se suponen objeto de intervención social por parte de un actor.

Es relevante mencionar, que surge a partir del intento de transformación social en el período de Allende en Chile, en el año 1972, donde se produjo una dispersión entre la planificación económica normativa (confinada en meros formulismos), la conducción económica y la conducción política que trajeron consigo serios problemas. Bajo esta perspectiva, la metodología PES persigue integrar esos tres aspectos $y$ puede ser aplicada en cualquier sistema de conducción en situaciones de conflicto, de cualquier grado o tipo, por su parte Matus (1987) asegura que es una planificación democrática.

Alizo (2020) plantea que la planificación de situaciones está conformada por dos vertientes principales, el abstracto formal y el histórico concreto. El primero consiste en el conocimiento detallado de la realidad objeto de estudio, en el que se detecta el origen de las diferentes situaciones y se explica todo el comportamiento del sistema a través del análisis de un conjunto de teorías complementarias, que buscan dar respuesta a la acción y a la dinámica del movimiento situacional. Algunas de las teorías que se utilizan son: teoría social, del juego, del comportamiento, de la acción estratégica, de la probabilidad, de los escenarios, cálculo interactivo, entre otras.

El histórico concreto por su parte, ubica a la planificación dentro del contexto económico, político y social que viene enfrentando el sistema, de manera que se tenga un conocimiento general y específico de la dinámica y de los aspectos contextuales donde estará inmerso el proceso de planificación (Alizo, 2005). Con referencia a lo anterior, se tiene entonces, que la PES se sustenta de un marco teórico interdisciplinario y de la noción contextual del sistema, generando todo un modelo íntegro capaz de revertir situaciones conflictivas.

Vale la pena señalar, que los actores sociales en este proceso juegan un papel fundamental y esto se debe a que son los únicos productores colectivos de eventos, que en consecuencia, son considerados sujetos del cambio situacional. Alizo et al, (2006) sostienen que un actor es una persona 
Gutiérrez Silva, José Manuel; Romero Borré, Jenny; Hernández Fernández, Lissette; Vega Jaramillo, Flor Yelena

Planificación estratégica situacional: Un proceso metódico-práctico

natural o jurídica que tiene la capacidad de intervenir o incidir sobre la realidad de un sistema a través de la acción no rutinaria concertada.

Matus (1980), expone que las fuerzas sociales son fenoestructuras complejas debido a que conforman acumulaciones de ideologías, creencias, valores, proyectos, futuros, peso político, peso económico, liderazgo, organización, información, control de centros de poder e influencia sobre otras fuerzas sociales. De esta manera, el actor social tiene la ventaja de tomar decisiones acerca del rumbo del entorno de un determinado sistema, pues es él, quien se desplaza en el mismo con factores de tipo socialcultural, elige la direccionalidad de los eventos, apoyado en la acción propia y, puede y debe unirse a la intención que tengan otros actores.

Es por ello, que la PES tiene validez para formaciones sociales donde se presenten algunos de los siguientes problemas que enumera Matus (1987:1) La reproducción del sistema es conflictiva; 2) algunas fuerzas sociales de peso actúan con el propósito de reforma o transformación del sistema; 3) las relaciones del sistema planificado con otros sistemas es conflictiva; 4) los propósitos de los actores que planifican se definen como algo más que meros logros económicos medibles en términos de contabilidad nacional; 5) se reconoce la existencia de oponentes en lucha; 6 ) los actores no son homogéneos y los oponentes pueden estar al interior de un mismo actor; 7) resulta ineficaz la mera planificación económica y surge como necesidad la integración de la planificación política.

En resumen, se tiene que el modelo de planificación estratégica situacional creado por Matus, es un proceso que precede y preside la acción, en el cual el punto de partida es la `situación inicial', representada por un problema que se expresa en un diagnóstico de interés para el actor que desea cambiar y encaminar estas dificultades hacia la dirección que se quiere lograr, mejor conocida como 'situación objetivo', que muestra el punto de llegada y trabaja como guía direccional u horizonte de la estrategia, de la imagen que se anhela alcanzar.

\section{Momentos de la planificación estratégica situacional}

De acuerdo a Matus (1987) el modelo PES no aborda el concepto de etapas como la planificación tradicional, sino que considera el análisis $y$ procesamiento de problemas y no de sectores, se utiliza por tanto, el concepto de 'momento', entendido como la instancia, ocasión, circunstancia o coyuntura, por la que recorre un proceso que no tiene principio ni fin. Matus (1987) revela que en planificación de situaciones los momentos se encadenan y cierran circuitos repetitivos para ayudarse mutuamente y puedan culminar en uno distinto de ellos. Representan un proceso continuo en cadena.

Los momentos a los que se hace referencia son denominados de la siguiente manera: Momento explicativo (M1), Momento normativo (M2), Momento estratégico (M3) y Momento táctico-operacional (M4). Cada problema expuesto en el plan y el plan como tal, debe desarrollarse pasando por estos momentos, que se dan de forma continua, en cualquier orden y en la que 
cada momento considera a los otros (Matus, 1987; Gutiérrez et al, 2016). En la Tabla 1, presentada a continuación, se puede observar la manera en la que trabajan los momentos en este método.

\section{Tabla 1}

Momentos en la planificación estratégica situacional

\section{Problema 1: M4 $\rightarrow \mathrm{M} 1 \rightarrow \mathrm{M} 2 \rightarrow \mathrm{M} 3$ \\ Problema 2: $\mathrm{M} 2 \rightarrow \mathrm{M} 3 \rightarrow \mathrm{M} 4 \rightarrow \mathrm{M} 1$ \\ Problema 3: $\mathrm{M} 1 \rightarrow \mathrm{M} 2 \rightarrow \mathrm{M} 3 \rightarrow \mathrm{M} 4$ \\ Problema 4: M3 $\rightarrow \mathrm{M} 4 \rightarrow \mathrm{M} 1 \rightarrow \mathrm{M} 2$}

Fuente: Elaboración propia a partir de Matus (1987).

Con esta ilustración, se evidencia la interacción de los momentos con los problemas y como estos se comportan sin alterar algún orden. El proceso continuo que lo caracteriza sistemáticamente, rompe el concepto de etapa en secuencia lineal con la que trabaja la planificación normativa, accediendo a un diseño de modelo novedoso que facilita la labor de cambios situacionales.

\subsection{Momento Explicativo: M1}

El momento 1, denominado momento explicativo, toma como palabras clave el 'fue', 'es' $y$ 'tendería a ser' del objeto planificado, haciendo énfasis en el pasado, presente y previendo el futuro de los acontecimientos. En este momento se explican todos los acontecimientos y problemas que existen en la realidad de un sistema, esta explicación es denominada diagnóstico situacional y es de insumo para el sujeto planificador y su posterior análisis situacional. Matus (1987) señala que entender la situación inicial tiene el significado de reconsiderar la validez del conjunto de problemas relevantes que se obtiene con la visión de síntesis del sistema que los produce. Pues, unos problemas son consecuencias de otros, que a su vez, causan, refuerzan o aminoran los primeros, diagrama 1 . 


\section{Diagrama 1 \\ Momento Explicativo de la PES}

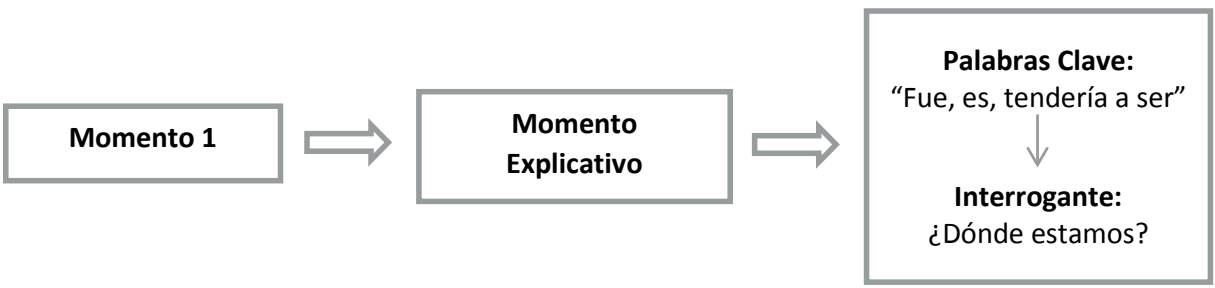

Fuente: Elaboración propia a partir de Matus (1987).

De esta manera, el momento se enfrenta a la interrogante ¿Dónde estamos?, dando pie al Análisis Situacional del Problema, que es una técnica sistematizadora de las causas de un problema sobre una realidad (Zambrano, 2006); obliga a adelantar soluciones y reconocer que ese problema puede ser explicado desde diversos puntos de vista por los actores en contacto con él. La técnica culminará con la expresión gráfica del Flujograma Situacional.

Sin embargo, antes de la realización del flujograma, se encuentra el Análisis FODA y la Matriz de Jerarquización de los Problemas. EI primero es definido por Thompson et al, (2012), como la herramienta usual para realizar el diagnóstico de una organización o sistema, compuesto por las variables: Fortaleza, Oportunidades, Debilidades y Amenazas; permite evaluar las competencias y carencias, tanto a nivel interno como externo, del objeto planificado. Zambrano (2006), agrega que el FODA conlleva a entender mejor cuáles son los factores endógenos y exógenos que influyen favorablemente o desfavorablemente en el desempeño de la institución, que condicionan la posibilidad de llevar a cabo su misión, visión, objetivos y metas.

Luego que se efectúa este análisis, queda claro el contexto situacional del sistema y se procede a detectar los principales problemas que agobian al objeto planificado. Por lo que se realiza a diferentes actores vinculados directamente con el objeto, la matriz de jerarquización, que permite organizar las debilidades y amenazas de mayor a menor, según la puntuación que le den los actores. Los valores estarán comprendidos entre 0 y 2 , entendiéndose el ' 0 ' como un valor más bajo y el ' 2 ' como la puntuación más alta. Las variables que se analizarán considerando las debilidades y amenazas son las siguientes (Alizo, 2005):

- Gobernabilidad (G): Criterio que define si el actor posee o no control sobre el problema.

- Tiempo de Maduración de los Resultados (TMR): Elemento que da a conocer el tiempo probable de conseguir un cambio representativo de los objetivos propuestos. 
- Costo de Postergación (CP): Es el costo político, económico y social en el que incurrirá el problema si no es resuelto lo antes posible.

- Valor Político (VP): Se refiere a la importación o valoración que tiene el problema para el actor social que planifica, su partido político y la sociedad en general.

- Impacto Político (IP): Consiste en la contribución del plan en el problema, considerando los tres cinturones de gobierno: Proyecto, Gobernabilidad y Capacidad de Gobierno.

- $\quad$ Recursos (R): Es la disponibilidad o no de recursos económicos, políticos, humanos, organizativos e instituciones que posee el sujeto planificador para hacer frente al problema.

- Innovación obligatorios en el problema, sobre la base de incorporación o introducción de alguna novedad o al perfeccionamiento de los conocimientos técnicos, teniendo como referencia que existen otras dificultades que sólo requieren continuidad y corrección, por lo cual no requieren la incorporación de innovación.

Cabe destacar que cada uno de estos indicadores se responderá con una perspectiva de poder en la toma de decisiones del objeto planificado, con el fin de revelar, cuál es el problema que incide directamente en el desarrollo eficaz de las actividades de la organización, permitiendo realizar una síntesis del análisis situacional $y$, construir en base a lo estudiado, el flujograma situacional.

Según Alizo (2005), el flujograma es una representación gráfica detallada sobre la atención de cada problema en particular, ya jerarquizado de acuerdo a su prioridad, significando el método de explicación situacional. Compone un análisis de esclarecimiento de los causales del problema, que sistematiza la reflexión de los factores intrínsecos y extrínsecos que provocan el surgimiento, reproducción y permanencia de dicho problema.

Este flujograma, estará compuesto por tres niveles situacionales, integrados por nudos explicativos que conforman la sistematización del problema por parte de los actores que realizan el proceso de planificación (genoestructura, fenoestructura y fenoproducción), el vector descriptor (VDP) que no es más que el acuerdo sobre el problema que se va a discutir, y las consecuencias o efectos del problema sobre el sistema (Matus, 1987). El cuadro 1 muestra un resumen de los principales elementos que componen el flujograma situacional, esencial en el proceso de planificación estratégica. 


\section{Cuadro 1}

Modelo del Flujograma Situacional del Problema

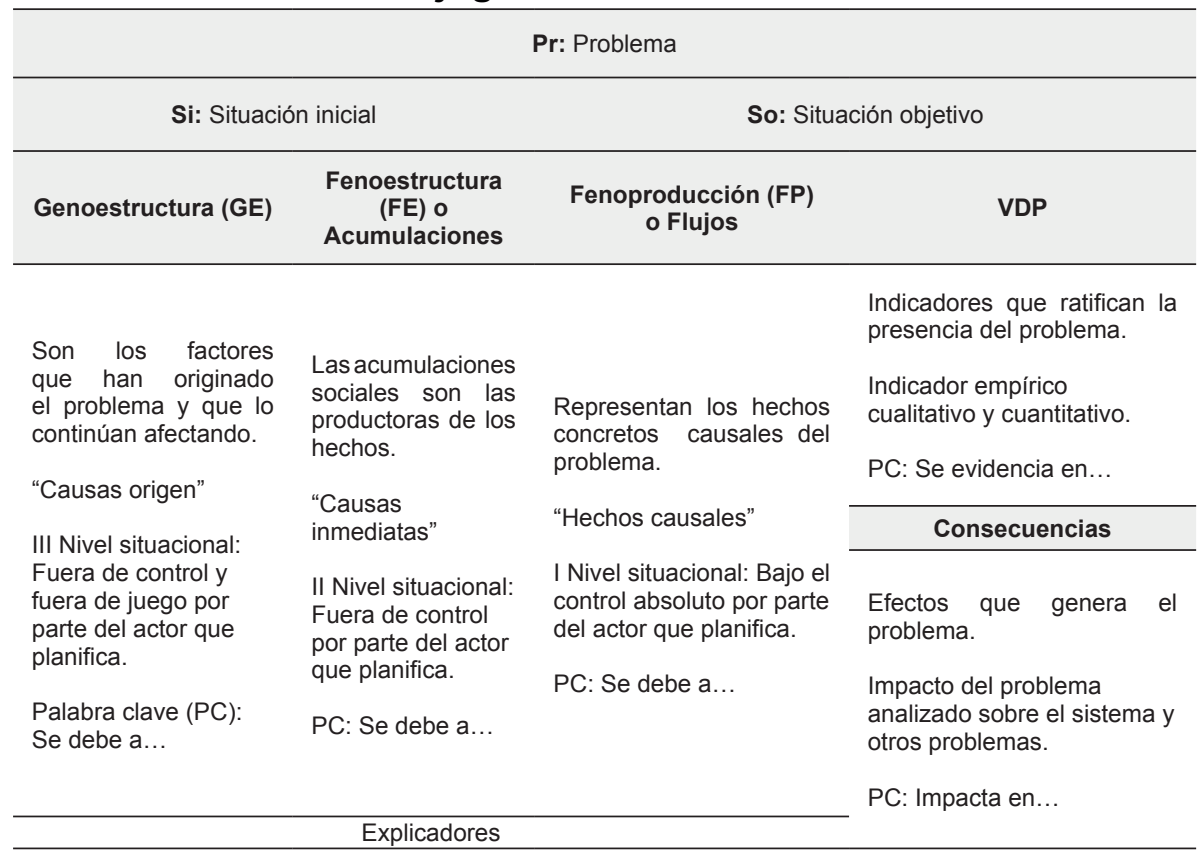

Fuente: Elaboración propia a partir de Alizo $(2005,2020)$.

Zambrano (2011) resume que la elaboración del flujograma situacional llevará a que el sujeto planificador actúe y enfrente los problemas causados de manera eficiente y efectiva, teniendo en cuenta que actuar sobre un aproximado de 25 causas supondrá un gran esfuerzo de carácter económico, político, organizativo y cognitivo. Por ello, plantea la necesidad de analizar cuáles son, entre las causas, las denominadas críticas o claves (nudos críticos); una vez que estos se encuentren identificados, la solución del problema debería ser más fácil.

Este punto es conocido como el
Análisis de Sensibilidad, definido como la selección de los nudos explicativos que provocan un mayor efecto positivo (reducen significativamente el problema general objeto de estudio) sobre el marcador del problema o VDP. Los nudos explicativos que generan hipotéticamente un mayor efecto positivo, serán los seleccionados como nudos críticos del problema en cuestión.

Para conocer cuáles son específicamente los nudos críticos, se comienza a identificar en la Matriz de Modificabilidad las causas que están bajo el control absoluto del sujeto planificador, las que se comparten o están fuera de su 
control y las que sencillamente provienen del impacto de otros problemas con los cuales interactúa el problema analizado $\mathrm{y}$, por consiguiente, son causas que están fuera de juego.

Además, se requiere revertir la situación de cada nudo explicativo hasta convertirlos en nudos objetivos, originando así la Matriz de Nudos Objetivos, en la que se explica lo que se quiere lograr a través del cambio direccional. Posteriormente, se realiza la Matriz de Criticidad, donde se evalúa a través de criterios de puntuación (de 0 a 1) el impacto que tiene cada nudo explicativo con las soluciones que se plantean de los mismos (nudos objetivos), llevando a conocer los nudos que realmente son críticos en el problema y modificables por los actores que ejecutan el proceso de planificación (Alizo, 2005).

El siguiente paso será realizar el flujograma con los nudos críticos seleccionados, con enumeración de izquierda a derecha y de arriba hacia abajo. Alizo (2020) acota, que cuando se realice todo el proceso descrito, se debe tener en cuenta la ubicación de los nudos en los niveles situacionales, pues sí estos están ubicados en su mayoría en la genoestructura y la fenoestructura, se estará ante un problema más complejo de solucionar, recuérdese que es allí, donde radican las causas origen y mediatas, en efecto, requerirán de más tiempo, maduración y recursos para su solución.

No se quiere decir con esto, que los nudos deben estar todos en la fenoproducción porque será más factible solventar las dificultades del sistema; se debe conocer concretamente la capacidad que tiene el actor de intervenir y poder generar acciones estratégicas no rutinarias, para corregir la problemática del entorno que se está valorando.

Consecutivamente, se procede a desarrollar la Matriz Nudos Críticos - Indicadores, evidenciando los indicadores cuantitativos y cualitativos existentes sobre cada nudo crítico del problema, que sin duda alguna, pone de manifiesto la existencia de subproblemas que deben ser resueltos a la brevedad posible. Finalizando, con el Árbol del Problema y Árbol de Objetivos.

El árbol del problema lleva la misma estructura del flujograma, sólo que no se representa de manera horizontal sino vertical. Aquí se detalla a simple vista, la jerarquía simétrica de los problemas desde un sentido que va de lo general a lo específico, o bien, de la genoestructura a la fenoproducción. Por su parte, el árbol de objetivos devela los nudos objetivos de cada nudo crítico abordado (Zambrano, 2006). Es así como culmina el momento uno de la planificación estratégica situacional, dando paso al momento normativo de este proceso.

\subsection{Momento Normativo: M2}

Luego de conocer la situación a la que se enfrenta el sistema, el momento 2 toma protagonismo en el diseño de cómo debería ser la realidad en oposición a los problemas que se desafían. De esta manera, el llamado momento normativo trabaja bajo la palabra clave 'debe ser' y se ocupa de la interrogante ¿Hacia dónde vamos?, poniendo de manifiesto la viabilidad de las estrategias que se implementarán en la búsqueda de solventar las dificultades presentes en la realidad.

Matus (1987) define este momento como la instancia de diseño, en la cual se involucra el debe ser de la estrategia (M3), el cómo debe ser la decisión y la 
Gutiérrez Silva, José Manuel; Romero Borré, Jenny; Hernández Fernández, Lissette; Vega Jaramillo, Flor Yelena

Planificación estratégica situacional: Un proceso metódico-práctico

ejecución del plan (M4) y cómo debe ser la norma que sirve de referencia para explicar la situación inicial (M1). Los diferentes momentos se vinculan, se interconectan y forman parte del diseño del plan, que abarca diferentes niveles de generalidad y especificidad, diagrama 2.

\section{Diagrama 2 \\ Momento Normativo de la PES}

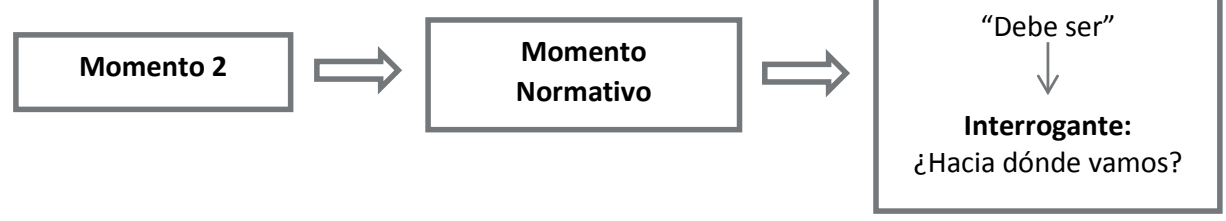

Fuente: Elaboración propia a partir de Matus (1987).

Alizo (2020) comenta que el momento 2 equivale al proceso que sigue la planificación normativa, consistiendo en la formulación del 'debe ser' $y$ para el logro de su producto terminal, requerirá la formulación de los Proyectos
Estratégicos de carácter direccional (Py's), debido a que son estas acciones, las que buscarán alcanzar la situación objetivo deseada. El diagrama 3 recoge la esencia del momento.

\section{Diagrama 3 Direccionalidad del Plan en el Momento 2}

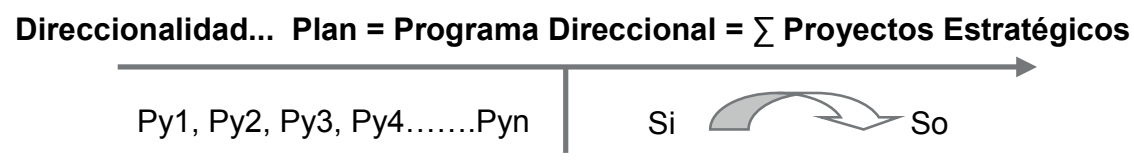

Fuente: Elaboración propia a partir de Matus (1987)

En el diseño del Plan que aborda la situación objetivo relacionada a precisar el alcance de la transformación del problema, se comienza con el Programa Base, se sigue con el Programa Direccional, para luego realizar la Matriz Pr's - Py's (Problemas
- Proyectos Estratégicos), Matriz de la Gran Estrategia, Matriz de los Actores Intervinientes, finalizando con la Carta Descriptiva o Módulos Operativos del Plan. Esto con el fin de tener bien definido el propósito del plan.

$$
\text { Matus (1987) precisa que }
$$


el programa es una oferta de enfrentamiento de algunos problemas, que sí tiene aceptación se convierte en un compromiso. Pero, la aceptación de la oferta por la base que tiene la legitimidad para ello, depende de su apreciación del valor de los problemas incluidos en la oferta y de la eficacia del proceso de intercambio de problemas que supone el camino propuesto por el dirigente.

Bajo este contexto, el Programa Base se cataloga como una declaración de convocatoria a la acción, es la oferta social donde los actores compiten por la adhesión de una base humana que quiere servir, pero también necesita sumar fuerza y legitimidad en torno a su contenido. En otras palabras, establece un compromiso del dirigente con la base social que intenta representar o con la organización que se propone dirigir (Matus, 1987).

El programa concibe la direccionalidad de cambio, expresando propuestas explícitas que coadyuvan al sujeto y la colectividad a alcanzar lo anhelado, incluyendo particularmente el Plan, que sigue el carácter de convocatoria, compromiso y guía para la acción práctica. Matus (1987) enfatiza, que el programa tiene que ser sincero y representativo, formulado con participación masiva de la población, con base a una direccionalidad previamente definida, con métodos uniformes, considerando los problemas, valor político y costos de los mismos, con coherencia, alcance, precisando proyectos estratégicos y garantizando acabar con las complicaciones que se presentan.

Lo anteriormente expuesto, describe parte de lo que sería el Programa Direccional, que constituye según Alizo (2005), el arco direccional que establecerá con exactitud los medios y recursos para lograr ciertos resultados de acuerdo a los escenarios previamente planteados por el sujeto planificador. Siendo necesario definir la situación objetivo y precisar los Proyectos Estratégicos de Acción de Carácter Direccional (Py's) suficientes para alterar la situación inicial (Si) y alcanzar la situación objetivo (So).

Una vez que ya se tengan definidos el conjunto de proyectos estratégicos (Py's) en concordancia con lo analizado en el momento 1 , se realiza la Matriz Pr's - Py's (Problemas - Proyectos Estratégicos), que permitirá medir el impacto que ejercen los proyectos estratégicos de acción de carácter direccional sobre los nudos críticos de los diferentes problemas a solucionar, la misma, identifica el nivel de afectación positiva o negativa, que ejerce cada proyecto sobre los nudos (Gutiérrez et al, 2016).

El sujeto o equipo planificador al evaluar cualitativamente a los proyectos, debe conocer el alcance de cada uno. Alizo (2005) deja claro que el proceso no es matemático, ni estadístico, requiere la experiencia sustentada en la intuición y el análisis lógico y consolidado sobre el balance del alcance de los efectos de la acción. Cabe destacar, que el proyecto que tenga más puntos positivos, será el adecuado para implementarse dado que reducirá drásticamente el problema.

Lo siguiente será abordar la Matriz de la Gran Estrategia o Matriz Cuantitativa de la planificación estratégica. David (1988) la cataloga como una técnica que permite a los estrategas evaluar cuantitativamente las estrategias alternativas con base a limitaciones y capacidades organizativas específicas, que requiere de decisiones anímicas para la asignación de ponderaciones y clasificaciones. Es por 
Gutiérrez Silva, José Manuel; Romero Borré, Jenny; Hernández Fernández, Lissette; Vega Jaramillo, Flor Yelena

Planificación estratégica situacional: Un proceso metódico-práctico

ello, que se debe utilizar con sabiduría y no indiscriminadamente.

Esta matriz conceptualmente determina, la atracción relativa de las diversas estrategias con base a factores internos y externos (debilidades $y$ amenazas) claves del objeto planificado, tomando en consideración el peso de los problemas de la Matriz de Jerarquización de los Problemas (Matus, 1987). De esta forma, se realiza un promedio ponderando con los valores totales obtenidos, lo cual permite determinar la importancia de cada debilidad y amenaza porcentualmente, visibilizando una pequeña comparación por proyectos, tabla 2.

Tabla 2

Modelo Matriz de la Gran Estrategia

\begin{tabular}{|c|c|c|c|c|c|c|c|c|}
\hline \multirow{2}{*}{ Problemas } & \multirow{2}{*}{ Peso } & \multirow{2}{*}{$\%$} & \multicolumn{2}{|l|}{ Py'1 } & \multicolumn{2}{|l|}{ Py'2 } & \multicolumn{2}{|c|}{ Py'n } \\
\hline & & & Calificación & Total & Calificación & Total & Calificación & Total \\
\hline \multicolumn{9}{|c|}{ Debilidades } \\
\hline \multicolumn{9}{|l|}{$D_{1} \ldots$} \\
\hline \multicolumn{9}{|l|}{$D_{2} \ldots$} \\
\hline \multicolumn{9}{|l|}{$D_{n} \ldots$} \\
\hline \multicolumn{9}{|c|}{ Amenazas } \\
\hline \multicolumn{9}{|l|}{$A_{1} \ldots$} \\
\hline \multicolumn{9}{|l|}{$A_{2} \ldots$} \\
\hline \multicolumn{9}{|l|}{$A_{n} \ldots$} \\
\hline Total & & $100 \%$ & & & & & & \\
\hline
\end{tabular}

Fuente: Elaboración propia a partir de Alizo (2005).

De esta manera, se le asigna una calificación cuali-cuantitativa a cada proyecto estratégico de carácter direccional propuesto, respecto al impacto que tenga sobre cada problema, es decir, sobre las debilidades y amenazas, de manera de evaluar si el proyecto incide positivamente sobre los diferentes problemas, reduciéndolos significativamente. La apreciación cualicuantitativa de cada proyecto estará comprendida por valores entre 1 y 4 (1: Muy bajo, 2: Bajo, 3: Alto, y 4: Muy alto), acotando que en el caso de las amenazas la escala oscilará entre 1 y 2 , ya que por ser factores externos al sujeto planificador, los niveles de afectación de los proyectos internos son menores.

Seguidamente, se realiza la Matriz de los Actores Intervinientes, que trabaja también en función de los proyectos y hace alusión a los actores sociales controlables y no controlables que intervienen activamente en el desarrollo de los proyectos estratégicos de carácter direccional (Zambrano, 2006). Con criterios de valoración, (0) si no interviene y (1) cuando el actor intercede, se sabrá fácilmente, la cantidad y cuáles son los principales actores que participan en los Py's. Teniendo hasta ahora seleccionado el proyecto idóneo y sus intervinientes para la solución de problemas.

Posteriormente, el trabajo del 
momento 2 se centra en elaborar los Módulos Operativos del Plan o la Carta Descriptiva. Matus (1987) asegura que la PES establece que el plan se exprese íntegramente en operaciones o módulos de acción, con el fin de garantizar la continuidad metodológica entre los lineamientos generales y los requisitos prácticos que propone la planificación. La teoría de las conversaciones como sistema articulado contempla 5 actos de habla ideales para la estructura del plan, respectivamente, tales como: las aserciones y apuestas, expresiones, declaraciones, directivas y compromisos de acción. Matus (1987) expone que el plan se compone centralmente de compromisos y complementariamente de declaraciones y directivas.

Las declaraciones, porque designa encargados o responsables del diseño y la ejecución de las propuestas que se incluyen. Las directivas, debido a que ordena a los designados por las declaraciones, a ejecutar y asumir determinadas obligaciones. Sin embargo, estos actos de habla mencionados, no constituyen el propósito terminal del plan situacional. Éste además está formado por operaciones, las cuales constituyen su razón de ser (Alizo, 2005), tabla 3.

Tabla 3

Modelo Carta Descriptiva

\begin{tabular}{|c|c|c|c|c|c|c|c|c|}
\hline \multicolumn{3}{|l|}{ Py's:... } & \multicolumn{3}{|c|}{ Objetivo General... } & \multicolumn{3}{|c|}{ NC Afectados $+\ldots$} \\
\hline Operaciones & Acciones & $\begin{array}{l}\text { Objetivo } \\
\text { Específico }\end{array}$ & Beneficio & Costo & $\begin{array}{l}\text { Res- } \\
\text { ponsa- } \\
\text { ble }\end{array}$ & Recursos & $\begin{array}{l}\text { Tiempo en } \\
\text { Ejecución }\end{array}$ & $\begin{array}{l}\text { Indicador } \\
\text { de } \\
\text { Monitoreo }\end{array}$ \\
\hline
\end{tabular}

$\begin{array}{ll}\mathrm{O}_{1 \ldots} & \mathrm{A}_{1} \\ \mathrm{O}_{2 \ldots} & \mathrm{A}_{2} \\ \mathrm{O}_{3 \ldots} & \mathrm{A}_{3} \\ \mathrm{O}_{\mathrm{n}} & \mathrm{A}_{\mathrm{n}}\end{array}$

Fuente: Elaboración propia a partir de Alizo (2005).

Por tanto, Alizo (2005) define el módulo operativo como una ficha visual gráfica, en la cual se trabaja bajo estructura modular e individualmente cada proyecto estratégico de acción direccional o simplemente, aborda, el proyecto adecuado elegido, que contiene información detallada sobre: los objetivos generales, objetivos específicos, operaciones, producto, acciones, beneficios, responsables por operación, recursos $\mathrm{e}$ indicadores de control $\mathrm{o}$ seguimiento. Los módulos operativos dan paso al momento estratégico de la planificación situacional, ya que este consiste en formular las estrategias, para lograr la viabilidad del plan o de los planes que pueden estar desagregados en programas direccionales, que a su vez contienen los diferentes proyectos 
Gutiérrez Silva, José Manuel; Romero Borré, Jenny; Hernández Fernández, Lissette; Vega Jaramillo, Flor Yelena

Planificación estratégica situacional: Un proceso metódico-práctico

estratégicos de acciones direccionales y procesales.

\subsection{Momento Estratégico: M3}

El momento estratégico de la planificación situacional se ocupa en esencia de la viabilidad del Programa Direccional del Plan
(M2), concentrándose justamente de acuerdo a Matus (1987), en el cálculo de articulación entre el 'debe ser' y el 'puede ser'. Sin embargo, no es un ajuste pasivo del 'debe ser' al espacio que permite 'el puede ser'. El debe ser es dominante y persistente en el conductor con voluntad de cambio, diagrama 4.

\section{Diagrama 4 \\ Momento Estratégico de la PES}

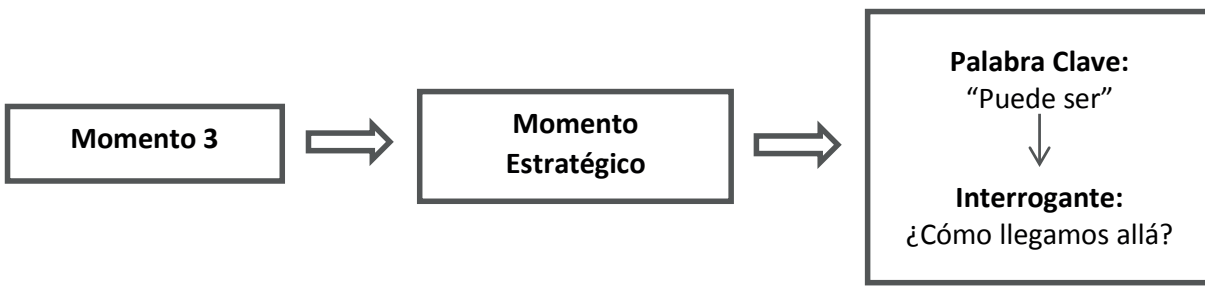

Fuente: Elaboración propia a partir de Matus (1987).

A pesar de que el momento 3 se preocupa por la viabilidad del programa, los otros momentos también se hacen presentes, con el análisis de viabilidad del arco de coyuntura en el momento táctico operacional (M4) y el análisis de lo que pudo o no pudo ser viable en la situación inmediata anterior (M1). Delgado (2007) comenta que es el momento del análisis de los actores, los que formulan, y los que se oponen, donde se mide tiempo, recursos y resultados, así como los escenarios posibles para la ejecución de las operaciones y demandas.

Alizo (2005), complementa que el momento busca crear posibilidades para la implementación efectiva de los proyectos estratégicos de acción relevantes, previamente seleccionados en los momentos precedentes. Asimismo, muestra que un proyecto de acción direccional será viable sí:

- Se puede tomar decisiones sobre su implementación o producción;

- Se puede hacer que opere eficazmente en la realidad, y

- Si su aplicación tiene una duración eficiente en relación a su propósito.

Además, se deben estudiar las restricciones situacionales y conocer las actitudes de los actores intervinientes en la ejecución del Py, pues los actores oponentes representarán restricciones situacionales. Alizo (2005) señala que estas últimas son aquellas barreras u obstáculos que limitan que un proyecto estratégico de acción direccional pueda implementarse en la práctica, por lo que es conveniente, precisar la relevancia y peso que tienen otros actores para la operativización adecuada del proyecto. Siendo necesario realizar la 
evaluación del Py a implementar según la metodología que evalúa los espacios direccionales.

De esta forma, se realiza la Matriz de Construcción de Viabilidad que es un instrumento gráfico que permite ilustrar sistemáticamente las posturas que asumen cada uno de los actores intervinientes, que mediante un esquema sinóptico se pueda establecer, con cierto nivel de exactitud y organicidad, la importancia, postura y participación de los actores, respecto al proyecto que se busca implementar (Gutiérrez et al, 2016).

En otro orden de ideas, se desarrollan las características específicas del proyecto estratégico de carácter direccional seleccionado, definiendo el objetivo general, para luego evaluar la motivación de realización del proyecto por parte de los actores, tanto controlables como no controlables. Para el desarrollo de esta matriz se utilizarán los criterios expuestos en el diagrama 5.

\section{Diagrama 5 \\ Criterios para la Matriz de Construcción de Viabilidad}

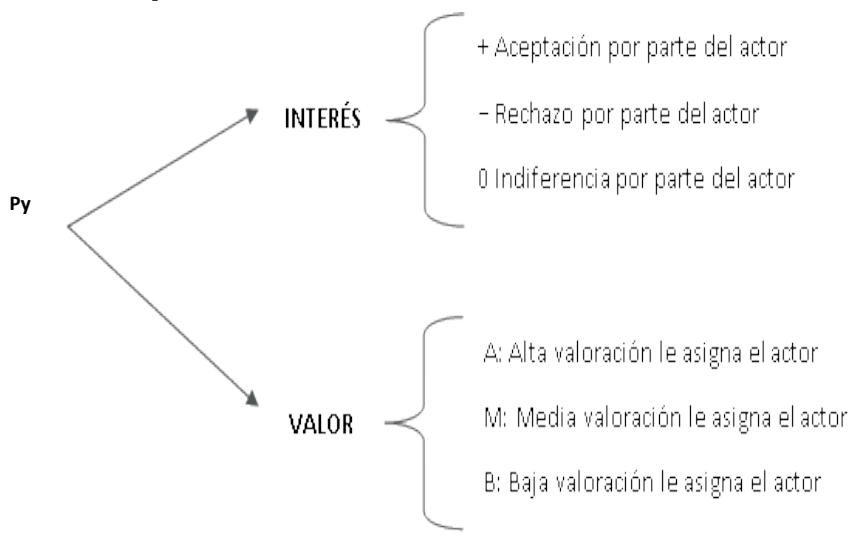

Fuente: Elaboración propia a partir de Alizo (2005).

Asimismo, se debe considerar el "Vector Peso", que consiste en especificar cómo el actor participa en la ejecución del Py en cuestión. En este sentido, se fija el porcentaje de intervención del actor en la implementación del proyecto, tomando en cuenta el 1) ROI: \% de participación del actor interviniente en la Capacidad Organizativa e Institucional; 2) RE: \% de participación del actor interviniente en el Financiamiento; y 3) RP: \% de participación del actor interviniente en la Toma de Decisiones.

El porcentaje total vertical para cada indicador mencionado (ROI, RE, RP) no puede exceder del $100 \%$, por lo que se distribuye este peso entre los diferentes actores de acuerdo a 
Gutiérrez Silva, José Manuel; Romero Borré, Jenny; Hernández Fernández, Lissette; Vega Jaramillo, Flor Yelena

Planificación estratégica situacional: Un proceso metódico-práctico

las consideraciones intervinientes que observe y estime el equipo planificador. También se realizará una sumatoria horizontal $(\Sigma)$ para cada indicador del vector peso, que al totalizarlos $y$ sumarlos verticalmente no exceden del $300 \%$. En la última columna, se observará el porcentaje de participación total por actor, que sale al comparar cada $\sum$ horizontal entre el peso total de $300 \%$, tabla 4 .

\section{Tabla 4}

\section{Modelo Matriz Construcción de Viabilidad}

\begin{tabular}{|c|c|c|c|c|c|c|c|c|}
\hline Py: & \multicolumn{4}{|c|}{ Objetivo General: } & \multicolumn{4}{|c|}{ NC Afectados + } \\
\hline $\begin{array}{l}\text { Actores } \\
\text { Intervinientes }\end{array}$ & \multicolumn{3}{|c|}{ Motivación } & \multicolumn{3}{|c|}{ Vector Peso } & & \\
\hline \multirow{2}{*}{ - Controlables } & \multirow{2}{*}{ Interés } & \multirow{2}{*}{ Valor } & \multirow{2}{*}{ Total } & \multirow{2}{*}{ ROI } & \multirow{2}{*}{ RF } & \multirow{2}{*}{ RP } & To & \\
\hline & & & & & & & $\Sigma$ & $\%$ \\
\hline
\end{tabular}

$A_{1}$

$A_{2}$

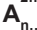

- No Controlables

$A_{1 \ldots}$

$A_{2 \ldots}$

$\begin{array}{lllll}100 \% & 100 \% & 100 \% & 300 \% & 100 \%\end{array}$

Fuente: Elaboración propia a partir de Alizo (2005).

Luego de realizada la matriz y observar los actores que no están de acuerdo con la implementación del proyecto, es necesario establecer una serie de estrategias que permitan negociar o trabajar sobre dichos actores, con el propósito de no presentar problemas a futuros. Matus (1987) asegura que cuando se devienen barreras situacionales, el planificador debe diseñar otras estrategias que busquen construir viabilidad a los proyectos estratégicos direccionales. En grandes líneas, las posibilidades son:

- Estrategias de cooperación con otros actores sociales, que suponen negociaciones para acordar vías comunes de acción, donde los actores ceden parte de sus intereses en beneficio del resultado esperado, como consecuencia de la acción concertada.

- Estrategias de cooptación, que implica que un actor social gane la voluntad de otros actores hacia la posición propia, en condiciones de apoyo.

- Estrategias de conflicto con otros actores sociales, que es la vía para dirimir el peso relativo de la fuerza de los actores con intereses encontrados frente a los proyectos 
en situación. Es decir, se tratará a través de enfrentamientos agotar las posibilidades del oponente.

De este modo, existen tres tipos de estrategias de construcción de viabilidad, cada una de ellas se materializa con la definición de acciones concretas procesales (Proyectos Estratégicos de Acción de Carácter Procesal) o Px's que pueden variar según Alizo (2005), entre: Mesas de diálogo o negociación, referéndum consultivo o revocatorios, reformas constitucionales, cambios en las legislaciones, enfrentamientos públicos y desacreditaciones, entre otras, que dependerán del estilo de gerencia que aplique el sujeto planificador.

El siguiente paso para finalizar, será tomar la misma matriz de viabilidad y anexar los proyectos estratégicos de acción procesal (Px's), monitoreando y evaluando de manera permanente el análisis estratégico ya que las actitudes y posiciones de los actores varían en el tiempo y por lo tanto, deberán modificarse las estrategias diseñadas.

Es así como su cumple el momento estratégico de la planificación, abordando todas las capacidades innatas que tenga el planificador para evaluar los actores oponentes al proyecto, que buscan intervenir de una u otra forma el cambio direccional del sistema. Por lo que el sujeto tendrá la iniciativa de pactar con los otros actores de modo que exista un beneficio colectivo o en la última instancia, aplicará la estrategia de conflicto, comentada con anterioridad.

\subsection{Momento Táctico- Operacional: M4}

Delgado (2007) manifiesta que en este momento se requiere de un sistema eficaz de seguimiento, monitoreo, control y elevados niveles de capacidad de recurso humano, técnico y político que asegure efectuar las demandas y operaciones del plan. Es en el M4 donde se está más cerca de la situación objetivo deseada y es el que comprende por ende, toda una síntesis del proceso de la PES, asegurando la realización del plan, diagrama 6.

\section{Diagrama 6}

Momento Táctico-Operacional de la PES

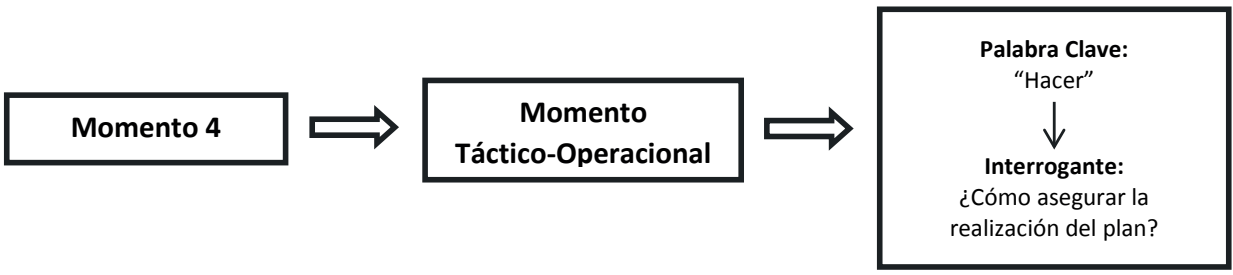

Fuente: Elaboración propia a partir de Matus (1987).

Alizo (2005) sostiene que el momento táctico - operacional comprende el cálculo que precede y preside la acción, es decir, lo que precede a la acción, es el conocimiento cuyo máximo nivel de desagregación lo constituyen los momentos anteriores (M1-M2-M3), y lo que preside la acción, 
Gutiérrez Silva, José Manuel; Romero Borré, Jenny; Hernández Fernández, Lissette; Vega Jaramillo, Flor Yelena

Planificación estratégica situacional: Un proceso metódico-práctico

es el monitoreo de las situaciones y cambios eventuales que se han producido en el sistema y que requieren la reestructuración del proceso.

Dado los criterios mencionados, la acción concreta se hace en el presente e impacta en el futuro. El futuro pesa como una prealimentación de planes prospectivos de distintos horizontes de tiempo, un mes, un año, una década, que traída a la mano en el presente permite encadenar direccionalmente lo que se debe y puede hacer hoy para alcanzar mañana los objetivos que se formulan. En algunos casos y dependiendo de la magnitud del problema, el futuro puede verse y sentirse en el corto, mediano o largo plazo.

Matus (1987) revela que todos esos plazos aportan una referencia direccional de distintos valores y significados para las decisiones del presente. Una vez concretada la acción decidida, otros factores influirán en la construcción de una nueva situación, que seguramente será distinta a la esperada. A partir de esta realidad, se debe recomenzar el proceso con una nueva explicación situacional y articulaciones de prealimentación y retroalimentación.

En resumen, el centro de la planificación estratégica tiene que ser un sistema articulado de discusiones y deliberaciones en los distintos niveles sociales, que produzcan como resultados, flujos incesantes de decisiones que precedan y presidan la acción, en la que el M4 disponga de todo un proceso de evaluación permanente, que verifique lo planificado y lo alcanzable con lo logrado en cada situación.

Una vez que se aborden estos puntos, se realiza el Sistema de
Dirección Estratégica compuesto de acuerdo con Alizo (2020), por los principios de gerencia pública moderna, así como el marco institucional que debe soportar el modelo de implementación de la PES. Si no se realiza, no se podrá conocer a los verdaderos responsables y coadyuvantes de construir los cambios propuestos y avanzar hasta el objetivo deseado.

Cabe mencionar, este sistema es creado por el Profesor Calor Matus y reseñado explícitamente en su obra "Adiós, Señor Presidente" (1994) y adaptado luego a "Los Tres Cinturones del Gobierno" (1997). El mismo Matus reseña que lo grandes errores que generan las crisis responden a fallas en la calidad de decisión sobre opciones críticas en épocas de normalidad, concibiéndose situaciones engorrosas producto de un sistema deficiente de planificación.

Dicho sistema de dirección estratégica representa el conjunto de dispositivos que estructuran prácticas de trabajo en una organización conducentes a la eficiencia, eficacia, reflexión, la creatividad, la responsabilidad, la apreciación situacional inmediata y la visión direccional de largo plazo, que descansa en un triángulo de tres sistemas clave (Gutiérrez et al, 2016); la Agenda de los dirigentes (A), que decide la lucha entre urgencias e importancias, e improvisación versus planificación; el Sistema de Petición y Rendición de Cuentas por Desempeño (B), donde se decide la lucha entre responsabilidad e irresponsabilidad; y el Sistema de Gerencia por Operaciones (C), donde se decide la lucha entre la gestión creativa o rutinaria (Alizo, 2005), diagrama 7. 


\section{Diagrama 7 \\ Modelo Sistema de Dirección Estratégica}

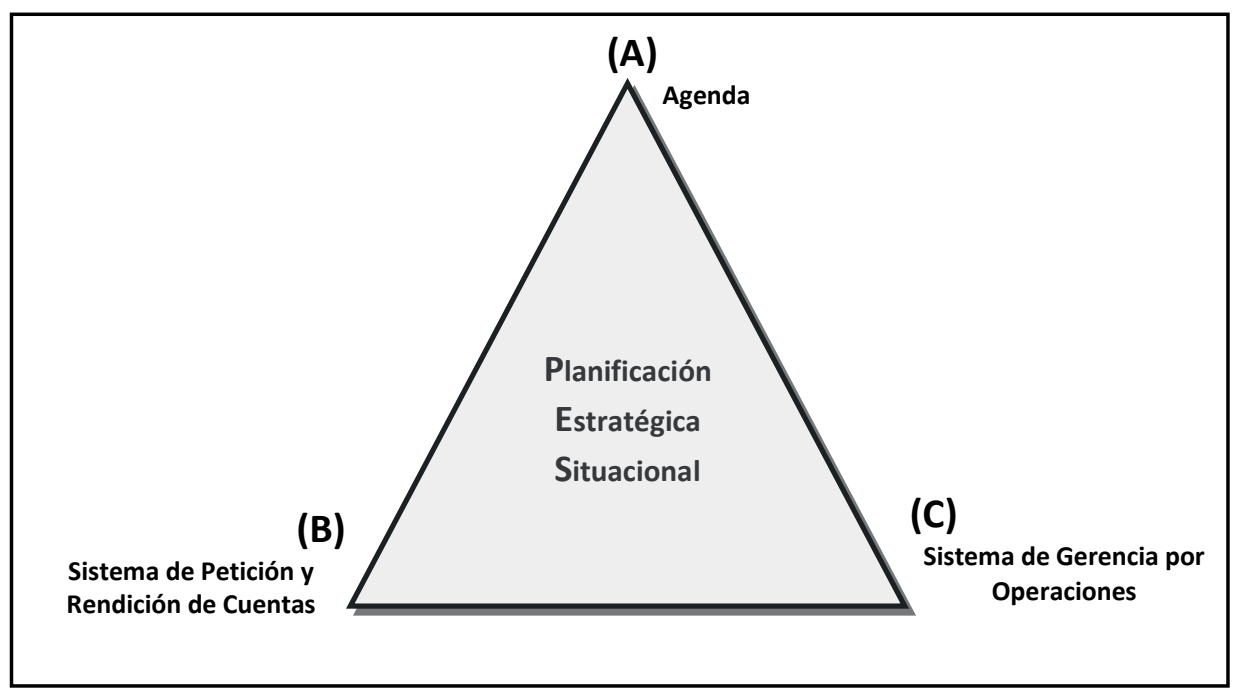

Fuente: Elaboración propia a partir de Alizo (2005).

La potencia de este triángulo reside en que si nadie es responsable de nada, no se rinden cuentas, ni nadie le cobra cuentas a nadie por su desempeño, entonces; a) la agenda no tiene defensas frente a la improvisación y las urgencias, b) la gerencia no tiene defensas para vencer la fuerza de la rutina y la apatía, y C) cualquier sistema destinado a elevar la calidad de la gestión sobra y es rechazado como un incompatible (Alizo et al, 2007).

Aunque no sólo basta con definirlo, es un gran peso el que tienen los actores sociales que conforman estas bases del sistema estratégico y tienen que velar por el verdadero cumplimiento de los objetivos deseados, se supone no se está conforme con la situación inicial del sistema y se ha planificado en torno a revertir tal situación, con apoyo de los proyectos estratégicos de carácter direccional y procesal.

La garantía del trabajo en equipo, el sentido de pertenencia de cualquier sistema, la visión compartida de logros, el beneficio colectivo y la intención de trabajar para obtener lo anhelado, llevará a la sociedad a conseguir lo que desea bajo un proceso de planificación estratégica que rompa con la sinergia de la incertidumbre y de no conocer lo que puede pasar si se sigue con las condiciones del presente.

\section{Consideraciones finales}

El modelo teórico-práctico de la planificación estratégica situacional a lo largo de los años ha concedido reconocer las necesidades de muchas organizaciones, sobre todo de carácter público y ha conseguido mantener una visión futurista de las mismas en el 
Gutiérrez Silva, José Manuel; Romero Borré, Jenny; Hernández Fernández, Lissette; Vega Jaramillo, Flor Yelena

Planificación estratégica situacional: Un proceso metódico-práctico

sentido, que busca determinar donde deberían estar en los próximos años y cómo debe ser gerenciada. Conocer los recursos necesarios para estar en esa visualización prospectiva, el conjunto de acciones a aplicar y los posibles resultados a obtener, dan una profunda tranquilidad a los gerentes.

Para ello, la planificación de situaciones requiere de cierta experticia, conocimientos formarles del arte de dirigir, intuición, mucho análisis y un basamento metodológico que guíe las acciones a alcanzar la situación meta u objetivo, donde los actores sociales son los principales protagonistas. Estos actúan y enfrenten los problemas causados de manera eficiente y efectiva, teniendo en cuenta, que actuar sobre las causas supondrá esfuerzos de carácter económico, político, organizativo y cognitivo que deben asumirse.

El método PES se enfoca en el plano estratégico del 'puede ser' $\mathrm{y}$ en el plano operacional de la 'voluntad de hacer', vigilando la direccionalidad de los objetivos esperados. Aquí interactúan como se mencionó, múltiples actores que pueden tener un comportamiento de alianzas, oposición e indiferencia, pero el actor que planifica está inserto y forma parte de la realidad del sistema y puede encontrarse con una contraposición a su plan por parte de otros actores. Ellos en su calidad de formar parte de la planificación, también tienen la potestad de elaborar planes, programas y proyectos que tiendan a coincidir, pues no existe un monopolio en la elaboración del plan, en tal caso entrarían en competencia.

Básicamente, es un proceso que está centrado en hacer posible los elementos que componen la planificación con una lógica de realización, dinámica de conducción y el aporte metodológico con expectativas, intereses, necesidades y problemas de las personas involucradas. Se debe seguir paso a paso el quehacer de cada momento como un proceso continuo en cadena para el análisis y procesamiento de problemas, accediendo a un diseño de modelo que no pierde vigencia y facilita la tarea de construir cambios situacionales. No obstante, la realidad actual de las organizaciones demanda trabajar con un sistema de planificación que combine varios métodos según sea la jerarquía y la complejidad de los problemas a abordar.

\section{Referencias bibliográficas}

\section{Alizo, M.A. (2020). Plan Reto. Alternativa de Sustentabilidad para la Venezuela del Siglo XXI. Editorial Académica Española.}

Alizo, M.A. (2005). Planificación Estratégica Situacional. Un Enfoque Teórico - Práctico. Venezuela, Escuela de Economía de la Facultad de Ciencias Económicas y Sociales de la Universidad del Zulia. Trabajo de Ascenso.

Alizo, M., Graterol, Á., Añez, S., y Ríos, M. (2006). Planificación situacional aplicada al sector socioeconómico venezolano. Caso: Emprendedores Emergentes de negocios. Revista Venezolana de Gerencia, 11(35), 352-382.

Alizo, M., Graterol, Á., Añez, S., y Socorro, C. (2007). Sistema Básico de Dirección Estratégico para Financiamiento al Proyecto EEN en el Municipio Maracaibo. Años 19992005. Revista Multiciencias, 7(3), $338-350$.

Basurto, X. (2016). Un acercamiento de la planificación estratégica y las organizaciones sanitarias. Dominio 
de las Ciencias, 2(3), 3-14.

Corredor, J. (2004). La planificación. Nuevos enfoques y proposiciones para su aplicación en el siglo XXI. Venezuela, Vandell Hermanos Editores.

David, F. (1988). La Gerencia Estratégica. Colombia, Legis.

Delgado, O. (2007). Sistema Nacional de Planificación y Presupuesto Participativo Venezolano. Venezuela, Alcaldía del Municipio Maracaibo.

Gutiérrez, J., y Colina, H. (2013). Estudios regionales para el crecimiento económico de Venezuela. Revista venezolana de Investigación Estudiantil, REDIELUZ, 3(1 y 2), 6675.

Gutiérrez, J. M., Alizo, M. A., Morales, M., y Romero, J. (2016). Planificación estratégica situacional: Perspectiva de una unidad científica universitaria. Revista Venezolana de Gerencia, 21(76), 607-626.

Martner, R., y Máttar, J. (2012). Los fundamentos de la planificación del desarrollo en América Latina y el Caribe. Textos seleccionados del ILPES (1962-1972). Chile, Naciones Unidas.

Matus, C. (1980). Planificación de situaciones. México, Fondo de
Cultura Económica.

Matus, C. (1987). Política, Planificación y Gobierno. Venezuela, Fundación ALTADIR, Instituto Latinoamericano y del Caribe de Planificación Económica y Social, ILPES.

Matus, C. (1994). Adiós, Señor Presidente. Venezuela, Fundación ALTADIR.

Matus, C. (1997). Los Tres Cinturones del Gobierno. Venezuela, Fundación ALTADIR.

Sabino, C. (2005). Diccionario de Economía y Finanzas. Venezuela, Editorial Panapo.

Thompson, A., Peteraf, M., Gamble, J., y Strickland, A. J. (2012). Administración estratégica: Teoría y casos. Décimo Octava. Editorial Mc Graw Hill.

Zambrano, A. (2006). Planificación Estratégica, presupuesto y control de la gestión pública. Venezuela, Universidad Católica Andrés Bello.

Zambrano, A. (2011). Planificación estratégica, presupuesto y control de la gestión pública. Venezuela, Universidad Católica Andrés Bello. 